\author{
Marie-Kenza BouHADDOU \\ Doctorante CRH-LAVUE \\ Ville et architecture \\ 204, rue du 4 Août 178969100 Villeurbanne \\ marie_bouhaddou@yahoo.fr
}

\title{
Les nouvelles pratiques artistiques et les organismes de logement social : entre service public, service marchand et lieux de créativité collective
}

Résumé : Cet article a pour objet de montrer, à travers l'analyse de deux cas lyonnais Prenez Racines! et $8 e$ Art l'oscillation entre service public et service marchand dans le cadre de nouvelles pratiques artistiques portées par un office HLM.

Dans la communication des organismes, ces pratiques font appel à une argumentation particulière centrée sur la notion de service.

En m'appuyant sur des éléments de définition et sur mes observations de terrain, je montrerai comment dans ces projets, la multiplicité des communications traduit la complexité d'un système d'acteurs et l'émergence de véritables lieux de créativité, d'altérité et de diversité tant artistique que sociale et urbaine.

Mots-clés : service public, service marchand, logement social, pratiques artistiques, communication, créativité

\begin{abstract}
New artistic practices and social housing organizations : between public utility, customers service and collective creativity places

Through the analysis of two artistic projects supported by a social housing organization in Lyon, France, this paper tries to show the fluctuations between public utility and customers' service.

In the organizations' public relations and communications, these artistic practices appeals a specific argumentation based on the idea of service.

Relying on definition elements and on my field's observations, I'll show how the multiplicity of communications express the complexity of the participants' system and allows the appearance of collective places of social, urban and artistic creativity, otherness and variety.
\end{abstract}

Keywords : public utility, customers services, social housing organizations, artistic practices, communication, creativity 
Depuis une quinzaine d'années, des organismes de logement social prêtent une attention toute particulière à des projets artistiques qui se situent sur les quartiers où ils possèdent leur patrimoine immobilier. Soit qu'ils fassent appel à des artistes soit que ceux-ci aient conduit un projet par exemple dans le cadre de la Politique de la Ville.

Ainsi, Prenez Racines ! est initié par la Maison des Jeunes et de la Culture Laënnec-Mermoz dans le cadre de la Coopération Culturelle de la Ville de Lyon tandis que $8^{e}$ Art est une commande publique portée par le bailleur social GrandLyon Habitat ${ }^{1}$.

De tels projets restant assez éloignés des préoccupations des bailleurs sociaux, quelles justifications donnent-ils alors pour les mener à bien?

Il s'agit de raisons relatives au vivre-ensemble, à la lutte contre la ségrégation urbaine et sociale, au maintien ou à la création de lien social et de la mixité, mais aussi à la valorisation du patrimoine et du cadre de vie. On peut toutefois supposer que les bailleurs, qui souffrent d'un déficit de visibilité, de reconnaissance et de légitimité, à la fois vis-à-vis de leurs partenaires techniques et politiques et de leurs résidents, puissent considérer ces pratiques artistiques comme de nouveaux moyens d'acquérir un rayonnement qui pourrait contribuer à faire évoluer leur image tout en élargissant leurs champs de compétences.

Dans la communication des organismes, ces pratiques artistiques, tout en occupant une place limitée, font appel à une argumentation particulière. Relevant en effet de compétences extérieures à leur «cœur de métier», cette communication exige des justifications appropriées, aussi bien en interne qu'en externe. Elle est en particulier centrée sur la notion de service que je propose d'analyser en détaillant les différents sens recouverts, leur articulation et leurs divergences. Mon propos montrera comment ces pratiques artistiques spécifiques menées dans des quartiers d'habitat social constituent ces quartiers en lieux émergents de créativité collective.

Je m'appuierai sur les observations menées dans le cadre de ma thèse : Logement social et nouvelles pratiques artistiques dont le sujet est inspiré par quatre années passées auprès de bailleurs sociaux en Rhône-Alpes. Elle est centrée sur l'étude des relations entre deux types d'acteurs : les organismes de logement social et les porteurs de nouvelles pratiques artistiques. Afin de cerner les différents sens que recouvre la notion de service, je m'attacherai dans un premier temps, à apporter des éléments de définition, tout d'abord pour ce qui concerne les

\footnotetext{
${ }^{1}$ Organisme de logement social du Grand Lyon
} 
expressions « service public », « service marchand » ainsi que les notions d' « intérêt général » et « intérêt particulier » qui s'y rattachent. Puis dans un second temps, au travers de des deux projets artistiques $8^{e}$ Art $^{2}$ et Prenez Racines ! $!^{3}$, je montrerai comment, d'une part, la multiplicité des communications traduit la complexité du système d'acteurs, et comment d'autre part, l'oscillation entre service public et service marchand exprime un contexte d'émergence de véritables lieux de créativité, qui placent ces quartiers périphériques au cœur d'un rayonnement qui les dépasse.

\section{Service, services}

\subsection{Service public et intérêt général, des notions intriquées}

Je définirai ici le terme de service, dans le but de comprendre les différences entre service public et service marchand et de saisir en quoi les projets que j'analyserai se situent dans une oscillation entre ces deux notions.

Le terme service est issu du latin servitium «condition d'esclave», «esclavage» puis «office ». Il s'agit à l'origine d'un mot du vocabulaire religieux et féodal qui désigne l'obligation de servir et l'action qui en résulte. Depuis le $19^{\mathrm{e}}$ siècle, service désigne un organisme chargé d'une fonction administrative. En économie, il est question d'une activité qui représente une valeur marchande sans correspondre à la production d'un bien matériel. Le terme est le plus souvent utilisé au pluriel (les biens et les services, la société de service etc.) ${ }^{4}$. Ainsi donc, ce mot recouvre plusieurs sens. $\mathrm{Si}$ «servir un dieu » (ou quelqu'un) relève d'un champ religieux ou d'utilité, « servir à » relève, lui, d'un champ commercial, d'usage et de fonction, tandis que « rendre service » apporte une dimension solidaire et désintéressée telle que l'on peut la retrouver dans l'expression « service public ».

Cette expression désigne, elle, l'institution, l'administration et la mission d'intérêt général. Dans le sens courant, elle a pris une acception plus large «d'obligation envers la collectivité $»^{5}$. Il s'agit d'une activité d'intérêt général exercée directement par l'autorité publique. L'expression peut aussi désigner l'organisme public qui est en charge de ce service. Il est question alors d'une «mission de service public » qui concerne des affaires régaliennes ou marchandes.

\footnotetext{
${ }^{2}$ Site Internet du projet $8^{e} A r t$ http://www.8e-art.com

${ }^{3}$ Site Internet du projet Prenez Racines! http://prenezracines.tb6380.com

${ }^{4}$ Alain Rey, Dictionnaire historique de la langue française, Le Robert, Tome 3, édition 2012, p. 3324 ${ }^{5}$ Id.
} 
Les activités comprises dans le service public sont considérées comme essentielles et stratégiques. Ouvertes à tous, elles échappent à la logique du marché et contribuent ainsi à la solidarité, la cohésion sociale, la culture et l'économie de la société.

Le caractère de service public contient outre une appréciation assez subjective, une dimension noble. Il s'agit d'un service gratuit qui se caractérise par la neutralité et la laïcité, une réserve dans l'expression des opinions individuelles dans laquelle l'intérêt privé s'incline devant l'intérêt général.

Pourquoi l'idée de service est-elle alors associée à des pratiques artistiques à priori exonérées de toute dimension d'utilité ?

Les pratiques dont il sera ici question se sont développées dans une filiation directe avec l'art contextuel, militant, social et activiste participatif, l'esthétique relationnelle, l'Outdoor Art et les Nouveaux Territoires de l'Art tels que décrits par Paul Ardenne, Nicolas Bourriaud, Fabrice Lextrait, Frédéric Kahn, Joëlle Zask, Hugues Bazin et Jean-Paul Fourmentraux (Ardenne, 2002 ; Bourriaud, 1998 ; Lextrait et Kahn, 2005 ; Zask, 2013 ; Bazin, 2006 ; Fourmentraux, 2012). Elles ne rejettent aucunement cette dimension sociale ou utile et sont d'ailleurs parfois appelées Useful Art ${ }^{6}$.

Cette notion de service public est étroitement liée à celle d'intérêt général. Le terme intérêt est un emprunt du latin classique interest «il y a différence entre », «il importe », interesse signifie «être entre », « être parmi », «être présent», « assister» et au figuré « participer à, s'occuper de $»^{7}$. Elle définit donc une relation entre un sujet (individu ou groupe) et d'autres personnes. L'intérêt est donc ce qui importe à quelqu'un, ce qui lui convient, lui procure un avantage et une utilité, au regard d'autres personnes.

L'expression «intérêt général » désigne, elle, les intérêts, valeurs et objectifs supposés être partagés par l'ensemble des membres d'une société. En France, ce sont les élus de la nation qui sont censés exprimer l'intérêt général.

L'expression se rattache d'ailleurs à celle de «bien commun » développée par Jacques Rancière, Joëlle Zask ou encore Antonio Negri (Rancière, 2000 ; Zask, 2011 ; Negri, 2010) dans le sens éthique d'un collectif partagé, politique, au-delà de la simple acception administrative et régalienne (Rancière, 2000).

Ces dimensions de la communauté et du public réfèrent aux thèses d'Hannah Arendt et de Jüngen Habermas (Arendt, 1995 ; Habermas, 1988). Il y a dans l'idée de public quelque chose de l'ordre de la publicité et de la visibilisation (Habermas, 1988). La communauté pour

\footnotetext{
${ }^{6}$ Paul Ardenne, «Rencontres Prenez Racines! », Musée Gadagne à Lyon, 29 mars 2013

${ }^{7}$ Alain Rey, Dictionnaire historique de la langue française, Le Robert,Tome 2, édition 2012, p. 1749
} 
Hannah Arendt est politique et altérité car elle traite «de la réciprocité d'être différents » (Arendt, 1995, 31). Par ailleurs, la dimension libératrice de l'art (des contraintes et de la domination) garantirait une dimension politique forte. Ainsi apporter l'art dans des quartiers où la domination sociale et économique s'exerce contribuerait à les inscrire dans une action politique (Arendt, 2001).

En politique, l'intérêt général (ou intérêt public) montre la finalité de l'action de l'Etat sans qu'il soit nécessaire d'en définir le contenu exact. Cela peut aussi bien décrire la somme de tous les intérêts particuliers qu'un intérêt spécifique à la collectivité qui transcende les intérêts individuels.

Il existe une distinction forte entre l'intérêt et les intérêts. En effet, l'intérêt désigne ce qui retient l'attention, ce qui est important, tandis que le pluriel traduit une idée de profit.

\section{2 Service marchand et services}

Par opposition à une notion de service, les intérêts sont fondés sur une dimension marchande et économique qui désigne des produits matériels ou immatériels.

Les services marchands font alors référence à des services non gratuits rendus par des entreprises moyennant paiement, à l'opposé des services non - marchands. Ils s'inscrivent donc pleinement dans une logique économique de marché.

Les services sont donc pris dans une triple pensée, utilitaire, fonctionnelle et économique.

En se rapportant aux pratiques artistiques, ils relèvent davantage d'un caractère d'usage et de fonction ou de revalorisation d'un cadre de vie. Ils sont supposés améliorer l'environnement des locataires des organismes de logement social.

Les offices HLM sont depuis plus d'une vingtaine d'années des entreprises privées, qui, bien qu'étant le plus souvent des $\mathrm{OPH}^{8}$ ou des $\mathrm{EPCI}^{9}$, n'en sont pas moins soumises aux lois du marché. D'autant plus depuis 2008, date à laquelle de nombreux offices se sont vus, sous des contraintes financières toujours plus drastiques, forcés de fusionner et d'affiner leur stratégie concurrentielle.

De fait, face à une conjoncture économique défavorable, les bailleurs des grandes agglomérations se retrouvent confrontés à une réflexion sur leur visibilité, leur identité et leur image, perspectives qui tant qu'ils étaient des émanations étatiques, leur étaient quasiment étrangères.

\footnotetext{
${ }^{8}$ Office public pour l'habitat

${ }^{9}$ Etablissement Public Commercial et Industriel
} 
Développant leur propre culture d'entreprise, ils doivent construire leur communication sur des spécificités qui pourraient les démarquer d'une autre structure. Ainsi GrandLyon Habitat a constitué un vocabulaire autour du développement durable, d'une part, et de la citoyenneté d'autre partir. Ces items trouvent leur traduction dans la communication des projets traditionnels mais aussi dans celle des projets artistiques.

Pour un bailleur social qui n'est pas supposé tirer profit de son entreprise, la notion de service marchand se rapporte donc davantage à ce qui peut servir à sa promotion et à sa visibilité. Il cherchera à conquérir de nouvelles «parts de marché », à agir sur de nouveaux terrains et à développer de nouvelles compétences. C'est dans ce contexte qu'il convient d'analyser l'implication et le soutien à des projets artistiques dans l'espace public. Par ailleurs, dans les projets artistiques, la position de payeur ou de propriétaire foncier des offices les place d'emblée dans une dimension marchande, avec des attentes en termes d'investissement, de profit et d'éventuels bénéfices ou pertes.

\section{Deux exemples : Prenez Racines ! et $8^{e}$ Art}

Mon choix s'est orienté sur deux des terrains observés dans le cadre de ma thèse. Bien que situés dans le même arrondissement de Lyon (donc supposément avec des constantes en termes de système d'acteurs, de problématiques sociales, urbaines et économiques), leur forme, leur esthétique, leur échelle et leurs enjeux varient fortement. En effet, l'un est initié dans un contexte d'événementiel culturel ${ }^{10}$, l'autre est un projet de coopération culturel élaboré avec les acteurs du quartier. L'un est une commande publique pilotée par la Direction Régionale des Affaires Culturelles et la Commission Nationale de la Commande Publique, l'autre est une expérimentation artistique co-construite avec les habitants dans le cadre des politiques de la ville. L'un peine à sortir du quartier malgré une volonté affichée de rayonner tandis que l'autre rayonne malgré une petite échelle et peu de moyens.

J'analyserai donc les écarts entre la communication d'annonce de ces projets et la réalité, et montrerai comment dans certains cas les pratiques artistiques, annoncées comme un service à valeur civique tendent vers une marchandisation.

\footnotetext{
${ }^{10}$ Lors de la candidature de Lyon aux Capitales Européennes de la culture, $8^{\mathrm{e}}$ Art avait alors été pensé comme l'un des projets concurrents.
} 


\section{$2.18^{e}$ Art}

Durant 2010 et 2012 j'ai été en charge du projet $8^{e}$ Art pour GrandLyon Habitat. Mes observations résultent de cette période d'immersion durant laquelle ont été lancées les consultations de la première série d'œuvres et des ateliers d'artistes, les études artistiques et celles du lieu ressource pour l'art dans la ville.

Bien que le projet soit placé sous la responsabilité de l'une des directions techniques de GrandLyon Habitat, je travaillais alors en étroite collaboration avec le président de l'office, qui en était l'initiateur, lui-même élu dans le $8^{\mathrm{e}}$ arrondissement de Lyon et conseiller à la Région Rhône-Alpes. Ce projet avant d'être celui d'un bailleur est donc celui de son président.

Ce qui caractérise le contexte d'émergence de $8^{e} A r t$ est un climat politique opaque entre la direction de l'office et la mairie d'arrondissement, et entre la mairie du $8^{\mathrm{e}}$, le Musée Urbain Tony Garnier ${ }^{11}$ et GrandLyon Habitat. S'ajoutent à ce contexte, une absence de clarification des acteurs du projet, notamment pour ce qui concerne la médiation culturelle et artistique, sous la responsabilité du bailleur mais non directement pilotée par lui et le rôle de décisionnaire de la D.R.A.C.

Je m'appuie donc à la fois sur mes notes de terrain et observations et sur l'analyse de documents produits par le service de communication du bailleur, d'entretiens avec la direction technique et d'interviews de son président recueillis dans la presse locale.

$8^{e}$ Art est une commande publique portée par le bailleur social GrandLyon Habitat, qui a débuté en 2007 à l'initiative du président de l'office. Elle vise à l'implantation de dix œuvres d'art contemporain le long du Boulevard des Etats-Unis, dans le $8^{\mathrm{e}}$ arrondissement à Lyon qui devront « revisiter l'utopie moderniste ${ }^{12}$. Ainsi partant d'une supposée «identité artistique » du quartier héritée de l'architecture et des fresques de Tony Garnier, $8^{e}$ Art devrait inscrire sa «demande d'art ${ }^{13}$ dans une continuité artistique, car selon le président de l'office, les

\footnotetext{
${ }^{11}$ Le Musée Urbain Tony Garnier a été crée en 1988 avec un groupe d'habitants, sous l'impulsion de GrandLyon Habitat et avec son soutien financier. Il a pour rôle de promouvoir le patrimoine moderne dans le quartier des Etats-Unis à Lyon et notamment de l'architecture de Tony Garnier et des fresques réalisées pour la plupart à la fin des années 1980. Il est vu par la mairie et GrandLyon Habitat comme un acteur incontournable de la vie du quartier des Etats-Unis.

${ }^{12}$ Consultation de la première tranche pour le projet de revalorisation artistique du Boulevard des Etats-Unis : $8^{\mathrm{e}}$ Art, mai 2010

${ }^{13}$ Emmanuel Hermange, «Comment faire advenir une demande d'art dans l'espace public ? », Zéro quatre ${ }^{\circ} 8$, printemps 2011
} 
habitants ont «tous été «formés » à l'expression artistique grâce au précédent créé par le Musée Urbain Tony Garnier $»^{14}$.

Par ailleurs, le projet d'art public s'accompagne de la création de dix ateliers d'artistes, vus comme des «fenêtres de l'art sur le quartier » ${ }^{15}$ et de la réhabilitation d'un « lieu ressource pour l'art ${ }^{16}$ afin que les artistes puissent $«$ travailler avec les habitants $»^{17}$.

Il s'agit pour le bailleur d'un projet complet qui mêle un art public légitimé à une médiation socioculturelle technique. C'est cependant ce maillage socio-culturel qui justifie l'implication de ses équipes techniques.

En interne, le bailleur adopte une terminologie technique et précise que $\delta^{e} A r t$ est pour lui le «projet de revalorisation artistique du Boulevard des Etats-Unis $»^{18}$. Avant la phase de médiatisation, j'ai pu noter qu'il arrivait au président de l'office de l'appeler « le projet des Etats » ou encore le « projet d'art sur le boulevard », signifiant ainsi, plutôt qu'une marque ou un logo, un ancrage territorial qui pourrait s'expliquer par une confusion entre son rôle de président et son rôle d'élu d'arrondissement. Le nom $8^{e} A r t$ a conjointement été choisi par le bailleur et la Direction Régionale des Affaires Culturelles sur la base d'un jeu de mots sur «art » et $« 8^{\mathrm{e}} »$ (pour le $8^{\mathrm{e}}$ arrondissement de Lyon). Il est intéressant de noter que tout comme l'autre projet phare d'art public de Lyon, Rives de Saône, l'ancrage territorial apparaît dans la désignation même des projets, même si pour $8^{e} A r t$, cet ancrage reste discutable.

Le logo du projet, son premier élément de visibilité, a été créé par une agence de communication lyonnaise ${ }^{19}$ choisie par le bailleur. Il est composé d'une étoile (évoquant le cinéma car le quartier est celui qui a vu naître les frères Lumière) du même vert que celui du logo du bailleur et du chiffre 8 traversé par une ligne qui représente le boulevard des EtatsUnis. Il a été décliné en plusieurs couleurs vives qui ne sont pas sans rappeler, quoique de façon très caricaturale, l'univers street-art. Il est cependant jugé simpliste et décevant par le public : « le logo $8 e$ art décliné (...) aurait pu échapper à une pauvreté déconcertante, loin des exigences formelles déployées par les artistes commandités $»^{20}$.

\footnotetext{
${ }^{14}$ Interview d'Yvon Deschamps, président de GrandLyon Habitat, dossier de presse $8^{e}$ Art, GrandLyon Habitat, décembre 2010

${ }^{15}$ Site internet du projet $8^{\mathrm{e}}$ Art $\underline{w w w .8 \mathrm{e}-\text { art.com } ~ « L e ~ m o t ~ d u ~ P r e ́ s i d e n t ~ », ~ Y v o n ~ D e s c h a m p s ~}$

${ }^{16} \mathrm{Id}$.

${ }^{17}$ Yvon Deschamps, discours inaugural de $8^{e}$ Art, Boulevard des Etats-Unis, 14 décembre 2010

${ }^{18}$ Cahier des Clauses Techniques Particulières, GrandLyon Habitat, mai 2010

${ }^{19}$ Agence Communiquez à Lyon.

${ }^{20}$ Emmanuel Hermange, « Comment faire advenir une demande d'art dans l'espace public ? », Zéro quatre $n^{\circ} 8$, printemps 2011
} 


\subsubsection{Un projet « citoyen » qui favoriserait l'urbanité}

Depuis peu, une dimension nouvelle, alors absente des propos du bailleur, apparaît : celle de la citoyenneté. Dans ses communiqués de presse, l'organisme se décrit maintenant comme un « créateur de confort citoyen ${ }^{21}$ lorsqu'il parlait deux ans auparavant de simple « dynamique citoyenne $»^{22}$, laissant ainsi penser qu'il ne s'agit pas du confort des citoyens, mais d'une citoyenneté qui émanerait du confort. On pourrait penser que le concept de citoyen relève d'une dimension politique, or il n'en est rien puisque l'illustration de cette phrase est que la structure réalise des programmes référents en matière de développement durable. La vision de la citoyenneté ne traduit aucunement la reconnaissance de la valeur des habitants en tant que porteurs d'expériences collectives et la désacralisation de l'expertise décrite par Loïc Blondiaux (Blondiaux, 2008) ; pas plus qu'elle ne recouvre les relations de réciprocité et de réversibilité dans une dimension égalitaire (Schnapper, 2000). Ainsi la définition qu'en donne le bailleur n'est pas liée à une construction sociétale qui pourrait se retrouver dans la notion contemporaine du « vivre ensemble » en tant qu'exercice de pratiques et de règles collectives. Même si, comme le souligne Catherine Neveu, la figure du citoyen est une double construction individuel et collective, il n'en reste pas moins que le citoyen est pris dans une dimension collective. Il est à la fois celui qui est « capable de s'extraire de ses appartenances et de s'exprimer dans l'espace public », et issu d'un collectif communautaire ou associatif (Neveu, 1999). Dans les deux cas, l'assemblage entre la dimension privée du confort et celle publique de la citoyenneté semble pour le moins curieux.

Pour autant, la position de l'office n'est pas claire, notamment pour ce qui est de la participation des habitants, une notion fourre-tout. En effet, le président ne veut rien de moins que «les gens se sentent heureux » en les rendant «acteurs du projet ${ }^{23}$. Mais la réalité est beaucoup moins généreuse car le «dialogue permanent entre les habitants, les acteurs locaux, le bailleur et les artistes »n'est en fait qu'un dialogue par intermédiaires interposés, dans lequel s'exercent une forte hiérarchie et une opposition entre experts et non experts. Ainsi, alors qu'il n'existe pas de lieux et de temps d'échanges avec les habitants, le président de l'office continue pourtant de parler de « dialogue riche et interactif $»^{24}$.

\footnotetext{
${ }^{21}$ Communiqué de presse de GrandLyon Habitat de l'exposition « Prendre place dans l'espace » réalisée dans le cadre des projets de médiation culturelle de $8^{\mathrm{e}}$ Art, 2 novembre 2011

${ }^{22}$ Dossier de presse du projet $8^{e}$ Art, GrandLyon Habitat, décembre 2010

${ }^{23}$ Extrait d'interview d'Yvon Deschamps pour Lyon Citoyen février 2011

${ }^{24}$ Dossier de presse du projet $8^{e}$ Art, GrandLyon Habitat, décembre 2010
} 
Pour GrandLyon Habitat, l'urbanité se décline à la fois dans une vision politicienne de l'espace public en tant qu'espace où prévaut l'intérêt général sur l'intérêt particulier, un espace neutre et laïque, un espace du consensus. L'urbanité est censée résulter d'une certaine vision de la citoyenneté en tant que respect de la règle et de la propreté. La notion de commun partagé voire négocié en est totalement absente. L'urbanité telle que l'entend le bailleur est donc assez loin d'une définition sociologique, telle que celle que l'on pourrait retrouver dans les travaux de Catherine Grout qui comprend «le mot urbanité dans une double articulation: d'une part, l'urbanité existe dans une réunion de personnes, avant que l'on ne parle de construit ou d'habitation, et d'autre part, elle existe parce que pour des personnes, il y a un monde en commun avant même l'institution de la société » (Grout, 2000, 83). La règle n'est donc pas pour elle la seule chose qui sous-tend cette communauté, mais bien un respect des altérités.

\subsubsection{Euvres d'art public et médiation : une démarche globale?}

La médiation est décrite comme le «point névralgique ${ }^{25}$ du projet, mais qu'entend-on par ce terme ? La médiation peut se comprendre dans une tentative de mise en relation de l'œuvre, de l'artiste et du public, autour de laquelle gravitent des notions telles que la traduction, l'intermédiation, la négociation le compromis ou la transaction (Akrich, Callon, Latour, 2006).

Bien que $8^{e} A r t$ soit présenté comme à la fois « artistique, esthétique, urbanistique, utopiste, créateur de lien social ${ }^{26}$. Il s'agit d'un projet d'art contemporain aux ambitions sociales et éthiques mais surtout d'un projet de visibilisation pour le bailleur car les actions en direction du vivre ensemble ou de la création de lien social sont pour ainsi dire inexistantes. Et ce, bien que le projet soit décrit comme «une dynamique citoyenne $»^{27}$, «source de lien social $»^{28}$, permettant de «créer les conditions d'un mieux vivre-ensemble ${ }^{29}$, qui favoriserait «la qualité de vie urbaine et collective ${ }^{30}$ et qui serait «l'élan d'une nouvelle vie ${ }^{31}$.Le rayonnement de $8^{e} A r t$ ne sert pas seulement le projet mais bien l'image du bailleur avec un argumentaire de l'ordre de l'unique, de l'emblématique et de l'innovation.

\footnotetext{
${ }^{25}$ Emmanuel Hermange, «Comment faire advenir une demande d'art dans l'espace public ? », Zéro quatre $\mathrm{n}^{\circ} 8$, printemps 2011

${ }^{26}$ Extrait d'interview d'Yvon Deschamps pour Lyon Citoyen, février 2011

${ }^{27}$ Dossier de presse du projet $8^{e}$ Art, GrandLyon Habitat, décembre 2010

${ }^{28}$ Interview d'Yvon Deschamps, dossier de presse $8^{e}$ Art, GrandLyon Habitat, décembre 2010

${ }^{29}$ Id.

${ }^{30}$ Dossier de presse du projet $8^{e}$ Art, GrandLyon Habitat, décembre 2010

${ }^{31}$ Interview d'Yvon Deschamps, dossier de presse $8^{e}$ Art, décembre 2010
} 
Tandis que les œuvres sont dites exceptionnelles, car elles apportent un «supplément d'âme $»^{32}$, « un nouveau souffle, un plus supplémentaire $»^{33}$, comme si le quartier était en déficit social, esthétique et éthique la médiation est, elle, du côté des habitants, perçus comme porteurs de ce déficit, du technique et du quotidien.

Peut-on se contenter d'une telle définition de l'œuvre d'art ? Cette dimension de « supplément d'âme » suffit-elle à traduire la complexité de la présence d'œuvres d'art dans l'espace urbain ?

Pour Catherine Grout, «l'art est ce qui réunit en un moment commun » sans toutefois que l'œuvre ne participe à l'édification du monde, elle permet cependant l'instauration de ce qu'elle nomme un « monde en commun » (Grout, 2000, 41).

Cette dichotomie apparente se résout si on analyse la nature des projets de médiation proposés, il apparaît en effet que tous s'inscrivent dans une vision traditionnelle de la réception culturelle puisqu'il s'agit d'expositions ${ }^{34}$, d'une chronique photographique ${ }^{35}$, de pièces de théâtre ${ }^{36}$, de spectacles de danse $\mathrm{e}^{37}$, d'ateliers pédagogiques ${ }^{38}$. Les habitants sont vus comme un public recevant de la culture et non comme un partenaire à part entière. On est donc assez loin de la construction partagée annoncée

Il s'agit pour le président de GrandLyon Habitat de lier « le beau et l'utile » dans une lecture platonicienne extrêmement simplifiée, le beau concernant sans doute les œuvres sur l'espace public et l'utile, les projets de médiation culturelle.

Dans le cahier des charges des études artistiques ${ }^{39}$, il a été demandé aux artistes d'imaginer un dispositif de médiation auprès des habitants, mais aucun d'entre eux n'a réellement pris en compte cette obligation contractuelle. Cela peut s'expliquer en partie par le fait que ce n'est pas le bailleur qui pilote le projet mais la D.R.A.C, laquelle a jugé que la médiation n'était pas

\footnotetext{
${ }^{32}$ Yvon Deschamps, «Rencontres Prenez Racines ! », Musée Gadagne de Lyon, 29 mars 2013

${ }^{33}$ Dossier de presse du projet $8^{e}$ Art, GrandLyon Habitat, décembre 2010

${ }^{34}$ Expositions itinérantes des maquettes de $8^{e} A r t$, ainsi que des photographies réalisées par les lycéens du quartier

${ }^{35}$ Il se passe quelque chose sur le Boulevard des Etats-Unis est une chronique photographique retraçant l'avant, le pendant et l'après projet sous forme d'un journal et de cartes postales distribuées aux habitants du Boulevard des Etats-Unis.

${ }^{36}$ Le N.T.H.8 (Nouveau Théâtre du $8^{\mathrm{e}}$ ) a proposé deux pièces de théâtre : Tomber la façade et $A$ fleur de béton, réalisées à partir de paroles d'habitants, recueillies par les acteurs auprès des résidents de GrandLyon Habitat concernés par les projets de Simon Starling Rotary Cutings et Bojan Sarcevic Traversée d'un ailleurs, puis jouées aux pieds des immeubles.

${ }^{37}$ Spectacles Lieux d'être de la Compagne Actes à proximité de l'œuvre Kiosk de Karina Bisch et sur l'œuvre d'Armando Andrade Tudela : 4 façades pour un patio.

${ }^{38}$ Les ateliers de l'association Art et Développement proposaient aux enfants de réaliser sur l'espace public des maquettes s'inspirant librement du travail de Karina Bisch sur les architectures emblématiques du $20^{\mathrm{e}}$ siècle.

${ }^{39}$ Cahier des clauses techniques particulières du projet de revalorisation artistique du Boulevard des Etats-Unis.
} 
l'affaire des artistes. Le bailleur s'est ainsi retrouvé en porte-à-faux avec la commune et les habitants car défendant un projet parachuté. Cette médiation culturelle s'est donc vue confiée à la Mission de Coopération Culturelle de la Ville de Lyon, par ailleurs souvent tenue à l'écart de l'avancement de $8^{e} A r t$ par le bailleur et la D.R.A.C. Ainsi, pour Kiosk, le premier projet $8^{e}$ Art à avoir été réalisé, l'artiste Karina Bisch devait utiliser les barricades pour communiquer durant la durée du chantier, mais elle n'a pas tenu à partager avec les habitants, le bailleur, les acteurs socio-culturels locaux et les élus. Par ailleurs, il n'y a eu, en tout et pour tout, qu'une seule réunion publique en présence de l'artiste ${ }^{40}$, durant laquelle elle a explicité son parti-pris esthétique devant une vingtaine de personnes dont plus de la moitié étaient des acteurs socio-culturels, des élus et des salariés de GrandLyon Habitat.

Pour donner une matérialité à cette «politique de médiation ${ }^{41}$, le bailleur, suite à une demande de la ville de créer des lieux de résidence artistique décide de livrer dix ateliers d'artistes à partir de containers recyclés ${ }^{42}$. Le président de l'office propose alors un rattachement avec $8^{e}$ Art en les implantant à proximité du Boulevard des Etats-Unis. Lors de la recherche de subventions pour réaliser l'opération, il est apparu que d'une part, le bailleur, règlementairement et techniquement, ne pouvait raccrocher ce projet de création d'ateliers à celui d'une revalorisation artistique (et donc d'un aménagement) et d'autre part, qu'il trouvait difficile de justifier auprès de son conseil d'administration un tel projet (car peu ou pas subventionné). C'est ainsi qu'il a été décidé d'intervenir au titre de l'accompagnement culturel et de la médiation. Néanmoins la confusion entre les deux projets persistait du point de vue du président de l'office qui voyait en partie ces ateliers comme la justification sociale du projet d'art public.

La notion de médiation est elle-même porteuse d'incompréhensions. Ainsi, pour les acteurs socio-culturels, elle consiste en un accompagnement social et culturel des populations ; pour le bailleur et pour la ville, il s'agit d'un outil de maillage du territoire, tandis que pour les artistes et pour les institutions artistiques, il s'agit de médiation artistique, au sens où Ruby l'entend, c'est-à-dire d'intermédiaire entre l'œuvre, l'artiste et le public (Ruby, 2005).

\footnotetext{
${ }^{40}$ Réunion publique $8^{e} A r t$, en présence de Karina Bisch, 11 février 2013

${ }^{41}$ Communiqué de presse de GrandLyon Habitat de l'exposition « Prendre place dans l'espace » réalisée dans le cadre des projets de médiation culturelle de $8^{\mathrm{e}}$ Art, 2 novembre 2011

${ }^{42}$ Consultation pour le projet d'installation de containers recyclés pour la création de 8 ateliers d'artistes à proximité du Boulevard des Etats-Unis (2 ateliers étant prévus en réhabilitation), GrandLyon Habitat, novembre 2012
} 
L'office parle des ateliers comme du «cœur d'une médiation vivante », mais si les ateliers sont le cœur d'une médiation vivante, les œuvres d'art le long du Boulevard seraient-elles mortes ou tout au moins figées?

Leur création a donné lieu à de nombreuses tensions, comme lorsque le projet a échappé au pilotage de la Mission de Coopération Culturelle de la Ville de Lyon qui était pourtant le commanditaire historique pour échoir à la D.R.A.C ou lorsque j'ai proposé la constitution d'un groupe de réflexion pour la réalisation du cahier des charges en vue du lancement d'une étude $^{43}$. J'ai alors vu se superposer les enjeux des différents acteurs comme la volonté d'associer cohésion urbaine et sociale et volet culturel pour la Région, le fait de pouvoir contrôler la légitimité artistique des candidats ou encore la volonté de maîtriser l'attribution des ateliers et de pouvoir réaliser une opération test afin d'entériner la réalisation de plusieurs dizaines d'ateliers dans le département par la D.R.A.C. Par ailleurs, la réalisation d'une opération à enjeux techniques (les ateliers devaient être réalisés avec des containers recyclés) d'une opération aux coûts maitrisés pour le bailleur et la nécessité de pouvoir choisir des artistes capables de faire participer les habitants par l'élue étaient aussi des données contradictoires. Enfin, le souhait de l'Ecole des Beaux-Arts de placer certains de ses étudiants ou encore la revendication de faire participer des artistes locaux et non «institutionnalisés » par les syndicats d'artistes venait clore ce millefeuilles de volontés paradoxales dont l'harmonisation semblait délicate. La D.R.A.C a ensuite quasiment dicté le cahier des charges technique au bailleur et a demandé que certains des acteurs ne soient plus invités. Dorénavant, elle se désintéresse du projet (qui peinait à trouver un financement et donc une viabilité économique) et que le groupe ne se réunissait plus. Malgré cela, GrandLyon Habitat a lancé l'étude pour la réalisation de ces ateliers et choisi un architecte, tout en sachant qu'il y a de faibles chances pour que ce projet voie le jour.

\section{1. 3 Communication visible et intrigue invisible}

Les outils utilisés par le bailleur visent à d'une partir, vulgariser, rendre accessible le projet aux habitants et d'autre part, à le faire rayonner à l'extérieur du quartier. Ainsi, en donnant

\footnotetext{
${ }^{43}$ A cette réunion participaient : la Région, la Direction Régionale des Affaires Culturelles, la médiatrice culturelle du projet $8^{\mathrm{e}}$ Art, le bailleur, l'élue à la culture de la mairie du $8^{\mathrm{e}}$ arrondissement, une représentante des Beaux-Arts et des représentants des syndicats d'artistes, mai 2011
} 
«une résonnance ouverte, internationale au projet ${ }^{44}$, notamment en choisissant «des signatures majeures de l'art contemporain », le bailleur (se) garantit un «projet de qualité » ${ }^{45}$. Cette volonté de légitimation se traduit par une profusion d'instances d'expertises et de validation, car GrandLyon Habitat a besoin de justifier le fait d'être commanditaire artistique. Il y a donc un «groupe d'experts ${ }^{46}$ composé de spécialistes de l'art contemporain, un « comité de pilotage » avec les acteurs techniques, un «groupe de réflexion sur les ateliers » et enfin un «comité technique de médiation » avec les acteurs territoriaux et culturels. Cette démultiplication d'instances témoigne d'une difficulté pour le bailleur à gérer la complexité de façon transversale, ce qui a pour conséquence une inertie dans la prise de décision et un manque de souplesse, alors que par ailleurs, le bailleur se présente comme porteur du projet (ce qui fonde son caractère innovant). Par ailleurs, il est à noter que le qualificatif de technique accompagne encore la médiation, tandis que ce qui est artistique à proprement parler, est l'affaire des experts.

En 2010, lors du lancement officiel du projet ${ }^{47}$, l'espace public et les tramways ont été physiquement marqués par des kakémonos qui sont restés plusieurs mois collés au sol et sur les rames. Ce marquage exprime la revendication du bailleur d'être reconnu en tant qu'autorité publique non seulement en tant qu'acteur public mais en tant que dépositaire d'une dimension publicitaire ${ }^{48}$. Cette «prise de l'espace public»s'est d'ailleurs faite sans l'autorisation à la Communauté Urbaine, propriétaire des mâts sur lesquels étaient accrochés les calicots, ce qui a eu pour effet de provoquer une vive polémique sur la portée «publique » du bailleur par le légitime représentant étatique. Ce débat sur ce qui est public et ce qui ne l'est pas, ce qui fait ou non espace, se retrouve dans les thèses de Catherine Grout qui définit le domaine public par ce qui «transforme le monde en une communauté d'objets qui rassemble les hommes et les relie les uns aux autres » (Grout, 2000, 37). Ici, l'occupation de l'espace public reste factice. Les autocollants posés comme pour signifier cette conquête de l'espace public d'une part, sont arrachés au bout de quelques jours. Cela traduit la difficulté de communiquer autour d'un projet qui n'existe pas encore spatialement parlant. Cependant l'office déploie beaucoup de moyens pour signifier qu'il se passe quelque chose. Ce n'est donc pas un hasard si cette phrase est reprise par l'un des artistes de la médiation, le

\footnotetext{
${ }^{44}$ Interview d'Yvon Deschamps, Dossier de presse de $8^{e}$ Art, décembre 2010

${ }^{45}$ Id.

${ }^{46}$ Appellation donnée par la direction de GrandLyon Habitat

${ }^{47}$ Inauguration officielle du projet en présence des représentants de la Ville de Lyon, de la Communauté Urbaine, de la mairie d'arrondissement, de la Région, de la DRAC, Lyon 8 ${ }^{\mathrm{e}}, 14$ décembre 2010

${ }^{48}$ Pauline Guinard, «Un autre art pour une autre ville ? , colloque international « Marges et art contemporain », Centre Marc Bloch-CERASS, Berlin, 10-11 octobre 2013
} 
photographe Cyrille Weiner qui intitule son travail Il se passe quelque chose sur le boulevard, quand $8^{e}$ Art à ce moment là ne se résume qu'à des maquettes, à des articles de presse et une campagne de communication.

Depuis le lancement du projet, la direction de l'office communique sur la création de dix œuvres. Les deux premières ${ }^{49}$ ont été livrées, toutefois la troisième ${ }^{50}$ rencontre de sérieuses difficultés en termes de faisabilité technique et financière puisque l'artiste a plus que doublé l'enveloppe qui lui était allouée au départ de l'étude et la quatrième est au point mort, parce que l'artiste est occupé par d'autres projets ailleurs dans le monde, que la mise en œuvre suppose l'adhésion des habitants que personne n'a réellement consultés et que la faisabilité technique n'est pas encore testée. Il y a de plus, de fortes chances pour qu'il n'y ait que huit œuvres livrées. En effet, le bailleur était contraint d'effectuer une mise en concurrence du fait de son statut d'établissement public tandis qu'en réalité, le projet étant sous l'égide de la D.R.A.C, celle-ci avait choisi un commissaire artistique qui, bien avant le lancement de l'étude, avait proposé au bailleur une liste de huit artistes dits « pressentis » ${ }^{51}$. Ce détail a mis le bailleur dans une posture très inconfortable rendant difficile (et hypocrite) la communication sur les futurs candidats.

\section{1. 4 Une volonté d'accompagner le quartier par l'art}

Le bailleur justifie son intervention par sa responsabilité en termes de qualité, qu'il décline aussi bien d'un point de vue urbain qu'artistique car pour lui, « la vocation de bailleur social intègre une forte dimension humaniste, attachée au bien-être des résidents et qui se traduit par une exigence de qualité, y compris environnementale et esthétique, apportée au cadre de vie $»^{52}$. C'est cette vocation qui le conduirait à accompagner le quartier dans une démarche artistique.

Cet attachement à la qualité du cadre de vie mène GrandLyon Habitat à envisager de transformer un ancien local commercial ${ }^{53}$ en « lieu collectif de ressource, d'expositions et de rencontres autour de l'art au cœur même du quartier des États-Unis» ${ }^{54}$. Le lieu a d'abord été

\footnotetext{
${ }^{49}$ Karina Bisch et sa pièce Kiosk et Armando ANDRADE TUDELA et son œuvre 4 façades pour un patio ont été livrées depuis le printemps 2013

${ }^{50}$ Bojan Sarcevic et son œuvre Traversée pour un ailleurs

${ }^{51}$ Entretien avec Patrick Rousseau, directeur du Patrimoine et de l'aménagement urbain, GrandLyon Habitat, 11 juin 2013

${ }^{52}$ Dossier de Presse du projet $8^{e}$ Art, GrandLyon Habitat

${ }^{53}$ Local du Bon Vigneron, rue Wakatsuki, Lyon $8^{\mathrm{e}}$

${ }^{54}$ Site Internet du projet $8^{\mathrm{e}}$ Art www.8e-art.com
} 
repéré par la Ville pour «ses » ${ }^{55}$ artistes en résidence qui en a fait la demande auprès de GrandLyon Habitat. Puis a été constitué un comité pour définir les enjeux mais aussi les modalités de gestion du lieu avec la Ville, la Mission de Coopération Culturelle, le responsable des ateliers de pratique amateur de la Ville de Lyon, l'architecte pressentie pour réaliser l'étude, des architectes urbanistes intéressés par la question de l'art dans la ville. J'ai demandé que soient associés des artistes mais ma demande s'est vue refusée par GrandLyon Habitat. A la suite de la première réunion, la ville a demandé que le Musée Urbain Tony Garnier soit aussi associé en tant qu'acteur culturel du quartier. Comme pour les ateliers d'artistes, j'ai vu ici se superposer des volontés contradictoires voire conflictuelles. Pour finir, devant l'absence de subventions (le budget de l'opération n'a pas été validé par la direction car jugé non prioritaire) le projet a été confié à la gestion d'un quatuor classique pour en faire des bureaux administratifs. Malgré cela, le président de l'office a de nombreuses fois communiqué sur la portée d'un tel lieu et continue de le faire sur le site Internet alors que le projet, en tant que lieu pour l'art dans la ville, est pour ainsi dire enterré.

Par ailleurs, depuis neuf ans, GrandLyon Habitat qui encourage et développe l'art dans la ville, se décrit comme «le premier bailleur social à avoir créé le concept des expositions artistiques » de ses locataires dans « des conditions réelles » pour «témoigner de la réalité de leur travail », opposant aux artistes reconnus institutionnellement aux artistes amateurs.

Enfin, cette prise en compte de l'art dans les nouvelles attributions du bailleur, bien qu'elle ne semble pas totalement assumée, est pourtant très visible, puisque le nouveau siège de l'office est doté d'une galerie d'exposition assez imposante qui masque quasiment l'entrée du bâtiment et brouille la lecture de sa fonction. Une passante s'interrogeait d'ailleurs récemment en désignant le siège social du doigt : « c'est quoi ça ? Une galerie d'exposition ! » ${ }^{56}$.

$8^{e}$ Art qui veut être une initiative citoyenne au service des habitants des quartiers se révèle un projet top-down dans lequel les résidents n'ont quasiment aucune place.

La volonté d'accompagner les transformations urbaines et sociales par des interventions artistiques du bailleur, sous des couverts humanistes visant à apporter un «supplément d'âme » à ce quartier, ne laisse en réalité aucune possibilité de participation aux habitants. La communication du projet traduit, une posture quelque peu équivoque dont la portée sur la valorisation du territoire semble évidente. En effet, en écartant les habitants du débat politique sur leur cadre de vie, il semble plus facile d'impulser un projet sans négociation.

\footnotetext{
${ }^{55}$ Les artistes intervenant en résidence dans le cadre de Mission de Coopération Culturelle (politique de la ville)

${ }^{56}$ Propos recueilli devant le parvis de la Gare de la Part-Dieu, en face du siège de GrandLyon Habitat
} 
Par ailleurs, GrandLyon Habitat utilise une communication qui le présente comme l'un des acteurs de l'innovation urbaine, sociale et esthétique et sert en fait davantage le bailleur que le projet en lui-même. Par ailleurs, il semble délicat de parler d'innovation sociale, urbaine, artistique ou éthique lorsqu'on parle d'art public.

Cette communication annonce un projet complet, justifié non seulement du point de vue artistique avec une mise en scène de l'expertise, mais aussi du point de vue social et éthique par son important dispositif de médiation. Toutefois ce dernier laisse apparaître de nombreuses failles et traduit encore une fois une volonté de ne pas réellement élaborer le projet avec les acteurs locaux et les habitants.

\subsection{Prenez Racines!}

Concernant le second projet Prenez Racines! j'ai mis en place plusieurs stratégies d'observation. D'abord sous une forme d'observation externe, j'ai choisi d'être présente pendant les temps forts, en ménageant des temps d'entretiens, plus ou moins formels avec les acteurs. J'ai aussi voulu être partie prenante du projet, d'une façon différente de celle que m'avait permise ma position professionnelle pour $8^{e} A r t$ en participant à un projet artistique connexe ayant pour sujet la pépinière créée par l'artiste : un film documentaire co-écrit avec la réalisatrice Sophie Fueyo ${ }^{57}$, ainsi qu'à une journée d'étude avec l'Ecole Nationale Supérieure de Paris ${ }^{58}$ sous la thématique des projets artistiques dans l'espace public.

Prenez Racines! prend place dans un quartier dit prioritaire puisqu'inscrit au titre de la Politique de la Ville depuis 1985, dont l'objectif est de «construire un projet de développement culturel qui tente d'accompagner les habitants dans la transformation urbaine $»^{59}$. C'est une expérimentation artistique démarrée en 2009 par l'artiste Thierry Boutonnier dans le cadre de l'opération de renouvellement urbain du quartier Mermoz dans le $8^{\mathrm{e}}$ arrondissement de Lyon. Dans ce contexte de transformations urbaines et sociales, qui prévoyait le relogement de la majeure partie des résidents, l'artiste propose la co-construction d'une pépinière urbaine d'une quarantaine d'essences d'arbres, plantées et soignées par des habitants. Ces arbres seront, en 2016, transplantés dans le nouveau quartier, tout comme les

\footnotetext{
${ }^{57}$ Aux arbres citoyens. Un essai poético-révolutionnaire, film documentaire réalisé par Sophie Fueyo, avril 2013

${ }^{58}$ Initiative menée à l'initiative de Pauline Guinard qui est enseignante au département de géographie à l'ENS

Paris et chercheuse au LAVUE-Mosaïque (thèse : Les espaces publics au prisme de l'art à Johannesburg.

Quand la ville fait æuvre de l'art et l'art æuvre de ville).

${ }^{59}$ Synthèse des Rencontres Prenez Racines! du 29 mars 2013, Musée Gadagne à Lyon, septembre 2013
} 
habitants y seront relogés. Dès 2007, et pendant dix-huit mois, l'artiste travaille en étroite collaboration avec la Maison des Jeunes et de la Culture qui l'accueille alors en résidence, à collecter auprès des résidents, comme un travailleur social le ferait, leur ressenti et leurs aspirations. Un an plus tard, avec le début de la rénovation urbaine, le plasticien fait quatre propositions : un rucher, une pépinière, un colombier et une bergerie. Ce sera finalement l'idée d'une pépinière qui sera retenue. Cette même année, l'artiste reçoit le prix $\mathrm{COAL}^{60}$, une reconnaissance de sa posture hybride entre art et écologie. D'autre part, le catalogue d'essences est réalisé avec les habitants. En 2011, la convention d'occupation est signée avec les parrains et marraines des arbres, le composteur collectif est mis en place et le règlement intérieur est rédigé et approuvé. En 2012, les parrains sont formés à la taille des arbres par l'association Les croqueurs de pommes, deux chantiers jeunes sont mis en route afin d'installer la cabane. En 2013, le composteur collectif est construit dans 1'Ecole Mermoz et les parrains sont formés à l'animation des jardins partagés par l'association Les Passe-Jardins, sur le principe de l'empowerment et du community organizing. (Allinsky, 1976, Freire, 2007). Depuis 2009, des fêtes, aux noms poétiques et évocateurs Tree Party, Pêches mignonnes ou encore Mermoz fait campagne ponctuent la vie de la pépinière et rassemblent les habitants autour de leur préparation.

A l'origine, Thierry Boutonnier avait choisi de nommer le projet Assolement pour Mermoz, en référence au partage de parcelles issu du vocabulaire agricole, mais le nom a été jugé trop pompeux par la Mission de Coopération Culturelle. La proposition Prenez Racines! suivie d'Expérimentation artistique à Mermoz a été choisie en remplacement. Toutefois l'artiste, les habitants et les acteurs techniques du projet parlent plus volontiers de « la pépinière ».

Tout en reprenant des éléments de langage issus de la culture hip-hop dans son logo, le projet n'a pourtant rien à voir avec cet univers. En effet, le logo, réalisé par l'artiste est réalisé sur une palette de récupération en bois, peinte en bleu et ocre, comme pour indiquer le ciel et la terre. Le mot «prenez » se déploie de la partie terre vers la partie ciel, tandis que le mot « racines » est centré dans la partie terre. La lettre «i » du mot « racines » est inversée, point vers le bas, comme si elle était plantée dans le sol. Le point d'exclamation, qui traduit l'injonction est lui peint en vert, pour rappeler le végétal. Le logo est taggué en noir et reprend les codifications du street-art comme l'écriture en continu ou l'exécution rapide.

\footnotetext{
${ }^{60}$ Prix délivré par l'association COAL (coalition pour l'art et le développement durable) dans le but de favoriser l'émergence d'une culture de l'écologie.
} 


\subsection{Un projet qui rend acteurs habitants et artistes}

L'artiste, qui parle volontiers de sa «volonté de créer un geste citoyen qui soigne son environnement et qui en récolte les fruits ${ }^{61}$, emprunte sa vision de la citoyenneté à la philosophie arendtienne dans sa relation entre politique et action (Arendt, 2001) et s'inscrit pleinement dans les projets artistiques participatifs décrits par Sébastien Gazeau et Françoise Liot (Gazeau, 2012 ; Liot, 2010).

Cette notion d'action, qu'il revendique se traduit par ailleurs par une arborescence de projets qui se raccrochent à Prenez Racines! Ainsi, plusieurs projets d'artistes et d'habitants viennent illustrer la dimension racinaire du projet chère à l'artiste comme la plantation d'un chêne de sept ans ${ }^{62}$ par l'artiste anglais Dan Harvey ${ }^{63}$ dans 1'objectif de rendre la ville plus verte constitue, comme le défendait Joseph Beuys, une véritable «sculpture sociale », un projet «écosophique» (Guattari, 1989) et «agriculturel»selon les termes de l'artiste lui-même ${ }^{64}$. Le film documentaire sur la pépinière Aux arbres citoyens! Un essai poéticorévolutionnaire ${ }^{65}$ explore, lui, la portée poétique et politique d'un projet co-construit avec les habitants, en proposant une lecture autonome. Après le visionnage du film, l'artiste s'est dit gêné par l'image bricolée que le film donnait du projet, en effet, le documentaire faisait du projet une lecture plus statique et moins complexe que dans la réalité. Ces actions artistiques plus ou moins directement liées au projet Prenez Racines !, en donnent une lecture, tout en cultivant leur propre indépendance et en développant de nouveaux réseaux et ramifications.

Je différencie ces projets à l'initiative d'artistes ou d'habitants de ceux impulsés par la maitrise d'ouvrage, comme cette autre réalisation connexe à Prenez Racines !, Eau de rose. Il s'agit d'un projet de réalisation d'eau de rose à partir de rosiers distribués aux habitants, ainsi que de la plantation d'un rosier remarquable sur un site à proximité de la pépinière en collaboration avec la maîtrise d'œuvre du projet d'aménagement du quartier. De façon concomitante, la Mission de Coopération Culturelle a proposé la signature de Prenez Racines!, ce qui a suscité une réaction vive de la part du responsable de la Mission Entrée

\footnotetext{
${ }^{61}$ Site Internet de Thierry Boutonnier : Domestication http://www.domestication.eu/

${ }^{62}$ Ce chêne est issu des glands plantés par Joseph Beuys (artiste britannique qui a fondé le plus grand parti écologique en Europe dans les années 1970) qui a planté un premier chêne à Kassel en 1982 qui a été suivi de 7000 autres dans les décennies suivantes.

${ }^{63}$ Du collectif artistique Beuys Acorn à Londres

${ }^{64}$ Entretien avec Thierry BOUTONNIER, artiste plasticien, 3 juin 2013, Lyon

${ }^{65}$ Sophie Fueyo a réalisé ce film que j'ai co-écrit, avril 2013
} 
Est $^{66}$. En effet, lorsque la médiatrice de la Maison des Jeunes et de la Culture a proposé que les parrains signent les bancs, il s'est alors écrié que les habitants avaient déjà assez participé et que c'était à l'artiste de signer son travail ${ }^{67}$. Eau de rose qui devrait sortir de terre pour « le symposium de la rose en $2015 \gg^{68}$ doit répondre à un besoin exprimé par la Mission Entrée Est de visibilité. Ce projet s'accompagne d'une expérimentation de pâture fleurie pour un cheptel de moutons, ainsi, durant deux semaines, un berger et son troupeau seront présents dans le quartier pour permettre aux habitants de « renouer avec la nature ${ }^{69}$. Cette pâture a été présentée aux élus comme étant fleurie sans toutefois s'attarder sur la nature de ce fleurissement, or en découvrant que les fleurs comestibles ne sont pas « un champ de $\operatorname{cosmos} \gg^{70}$, le responsable de la Mission de Coopération demande des explications ${ }^{71}$ car il attribue une valeur négative à l'image donnée par les fleurs destinées à être broutées.

Ces réalisations participent de la visibilisation de Prenez Racines !, mais contribuent aussi à chahuter sa dimension gratuite et solidaire, car le projet est perpétuellement en tension, entre macro et micro-action, être dans le faire et rayonner (et donc parler de et faire parler). En rayonnant, les projets prennent de l'autonomie et échappent aussi d'une certaine manière à une possible volonté de les ramener vers le propos par l'artiste qui décrit son travail comme une articulation entre le patrimoine vivant et le patrimoine bâti.

\section{2. 2 Une communication hybride calquée sur un système d'acteurs complexe}

Si $8^{e}$ Art utilise plutôt une communication institutionnalisée, Prenez Racines !, s'appuie sur une communication traditionnelle et explore aussi d'autres voies, notamment des procédés plus informels et organiques. La communication traditionnelle du projet se tourne vers plusieurs types de publics.

En premier lieu, il s'agit d'un public d'acteurs techniques, comme la Maison des Jeunes et de la Culture, le Centre Social, les techniciens de la Ville, de la communauté urbaine, et la maîtrise d'œuvre. Elle se décline sous la forme de mailing, newsletters envoyés par la Maison des Jeunes et de la Culture et Thierry Boutonnier, dans lesquels le ton varie entre propos poétiques et une certaine familiarité donnant l'image d'un projet partagé et convivial. Les

\footnotetext{
${ }^{66}$ La Mission territoriale Entrée Est est une antenne de la Politique de la Ville de Lyon qui intervient sur les thématiques suivantes : santé, emploi, insertion, habitat, gestion urbaine de proximité, économie, sécurité, prévention, jeunesse et égalité.

${ }^{67}$ Comité de pilotage de Prenez Racines !, Mission Entrée Est, Lyon, 30 septembre 2013

${ }^{68} \mathrm{Id}$.

${ }^{69} \mathrm{Id}$.

${ }^{70}$ Comité de pilotage de Prenez Racines !, Mission Entrée Est, Lyon, 30 septembre 2013

${ }^{71}$ Id.
} 
comptes rendus et dossiers de présentation prennent une forme plus conventionnelle et traduisent un «faire comme $»^{72}$ où l'artiste use d'un vocabulaire de l'entreprise ou des autres acteurs, de la même façon qu'il détourne au quotidien les attributs des travailleurs (casque, vêtements ou barrières de chantier ${ }^{73}$ ). Affirmant sa volonté de montrer que ce projet est un travail à proprement parler, Thierry Boutonnier me confiait d'ailleurs qu'il trouvait l'usage du terme « projet » inadapté pour Prenez Racines ! « on devrait dire travail plutôt que projet $»^{74}$. En deuxième lieu, il s'agit d'une communication conviviale avec des lettres rondes et des couleurs vives à l'égard des habitants ou des usagers du Centre Social. Elle est plus distanciée car elle s'adresse à un groupe d'usagers indifférenciés plutôt qu'à des personnes identifiées.

La communication est aussi largement vulgarisée comme c'est le cas lors des émissions de télévision $^{75}$ locales consacrées au projet. Il en va de même pour le film documentaire de

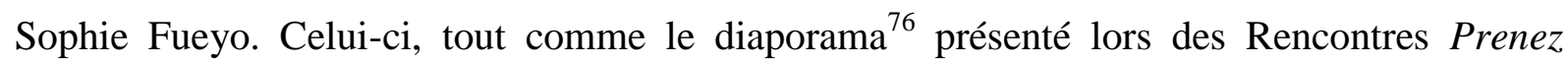
Racines! a contribué à valoriser les habitants qui auraient pu se sentir quelque peu étrangers au débat dans une telle instance.

En troisième lieu, il s'agit d'une communication institutionnelle interne qui présente le projet et ses évolutions au sein même des structures (structures socio-culturelles ou bailleurs sociaux). Elle est rapide, liste les projets et propose des rendez-vous sous la forme d'une newsletter adressée à l'ensemble des acteurs socioculturels, artistes et universitaires.

En quatrième lieu, il s'agit d'une communication vers les universitaires, comme lors des Rencontres Prenez Racines! ${ }^{77}$, ou lors des entretiens mis en place avec l'artiste Thierry Boutonnier $^{78}$. Elle vise à apporter un crédit et une légitimité scientifiques au projet et à l'artiste. Les Rencontres Prenez Racines! sous la direction de l'historien de l'art Paul Ardenne, rassemblaient l'ensemble des acteurs du projet (artistes, élus, bailleur social, acteurs techniques, universitaires, habitants, maitrise d'œuvre, maitrise d'ouvrage urbaine). Elles avaient pour objectif de retracer le contexte scientifique du projet et sa filiation, mais aussi de développer une justification artistique, politique et sociale par les porteurs techniques et financiers du projet. L'analyse de la synthèse des Rencontres, réalisée par la Maison des

\footnotetext{
${ }^{72}$ Stéphane Tonnelat, Séminaire «Lieux et enjeux », Ecole Nationale Supérieure d'Architecture de Paris Val de Seine, décembre 2012

${ }^{73}$ Lors de la fête de plantation du premier arbre du projet Prenez Racines !, 15 septembre 2011

${ }^{74}$ Discussion informelle après le comité de pilotage de Prenez Racines !, Mission Entrée Est, 30 septembre 2913

${ }^{75}$ Présentation de Prenez Racines ! lors du journal télévisé de France 3 régional ou l'interview d'Yvon Deschamps pour Lyon TV

${ }^{76}$ Réalisé par Sophie Fueyo à partir des paroles des parrains et marraines

77 « Rencontres Prenez Racines! », Musée Gadagne à Lyon, 29 mars 2013

${ }^{78}$ Entretiens réguliers que je réalisé avec Thierry Boutonnier qui portent essentiellement sur la définition des mots utilisés par les uns et les autres des acteurs.
} 
Jeunes et de la Culture et le Centre Social a permis de mettre en lumière d'une part, la visibilité de l'événement et d'autre part, le nombre et la diversité des participants «plus de 100 personnes ont assisté à cette journée : artistes, partenaires du projet Prenez Racines !, sociologues, urbanistes, étudiants, auditeurs, participants... ${ }^{79}$, traduisant ainsi, aux yeux des organisateurs, une forme de représentativité, condition de réussite (et de contrôle) des politiques de la ville.

En cinquième lieu, il s'agit d'une communication qui s'adresse aux acteurs des mondes de l'art dans le but d'une justification que l'artiste utilise comme un réseau complexe. Ainsi, lors des Rencontres Prenez Racines! la présence de Paul Ardenne pouvait difficilement passer inaperçue tandis que les services de la Direction Régionale des Affaires Culturelles en étaient absents. Le journal ForHum publié par Zone sensible ${ }^{80}$ est l'occasion non seulement de faire parler des travaux de l'artiste, mais aussi de figurer dans un réseau d'artistes et de chercheurs engagés aussi bien du point de vue social, qu'environnemental et éthique.

De même que le prix COAL a représenté pour l'artiste une approbation par ses pairs et surtout la reconnaissance du lien entre expérimentation artistique et environnementale. Enfin, le site Internet est aussi un moyen de rendre compte à la fois de l'actualité, et des différentes ramifications (Rencontres, mémoire d'étude, diaporama, émission télévisée etc.).

En dernier lieu, on peut observer une communication de justification des élus vis-à-vis de leur électorat, à visée politicienne. Ainsi lors des Rencontres Prenez Racines !, les élus ont inscrit le projet non pas dans une filiation artistique ou sociale mais bien dans un macro projet politique. Pour l'élu à la politique de la ville, «c'est d'abord un projet global qui engage l'ensemble des politiques publiques $»^{81}$, pour le conseiller de la Communauté urbaine, à la Mairie du $8^{\mathrm{e}}$ et à la Région et président de GrandLyon Habitat, qui intervient ici avec sa casquette politique, il incombe au politique de «créer les conditions d'intervention sur la ville », enfin, pour l'élue à la culture à la Mairie du $8^{\mathrm{e}}$ arrondissement de Lyon, Prenez Racines! est un outil de maillage du territoire.

\footnotetext{
${ }^{79}$ Synthèse des «Rencontres Prenez Racines ! » du 29 mars 2013, Musée Gadagne à Lyon, septembre 2013

${ }^{80}$ Résidence artistique collective en Seine Saint Denis d'artistes chercheurs travaillant sur le lien entre art et environnement.

${ }^{81}$ Louis Lévêque, élu à l'habitat, au logement et à la Politique de la Ville de Lyon, « Rencontres Prenez Racines !», Musée Gadagne de Lyon, 29 mars 2013
} 


\section{2. 3 Une communication informelle}

Pour ce qui est de la communication informelle, elle est de deux ordres : sous formes de discussions qui se font hors des cadres institutionnalisés de participation (moments de jardinage, les avant et les après réunions...) et sous la forme des jeux de langage dont l'artiste fait usage. En effet, il utilise le langage et la communication en tant qu'outil artistique, multipliant les jeux de mots, approximations et métaphores, jouant sur la dérision comme «langage des dominés » et les détournements. Ainsi il utilise non seulement les modes de faire de certains des acteurs, mais aussi leur langage, en précisant les acronymes «C.U.C.S : contrat urbain de cohésion sociale » et «A.N.R.U : agence nationale de renouvellement urbain » comme pour montrer sa maîtrise. Mais il fait aussi usage d'un vocabulaire scientifique rigoureux et précis comme lorsqu'il parle de l' «hydro-ensemencement», ou encore lorsqu'il nomme son projet Assolement pour Mermoz. Lorsqu'il dit qu'il s'agit de « catalyser un environnement transitoire grâce à la mise en valeur d'une biocénose urbaine et dépasse la seule animation sociale en contribuant à un «mieux habiter $»^{82}$, on mesure sa volonté non seulement de détournement mais aussi d'asseoir son expertise, qui n'est pas seulement artistique.

\footnotetext{
${ }^{82}$ Propos de Thierry Boutonnier dans la synthèse des «Rencontres Prenez Racines ! » du 29 mars 2013, Musée Gadagne à Lyon), septembre 2013
} 
Au travers de l'analyse de ces deux projets, des documents de communication des bailleurs, et des discours officiels des collectivités, s'est esquissée l'oscillation entre service public et service marchand.

En effet, ces deux projets qui prennent naissance dans le même quartier, soutenus par le même bailleur social, auraient pu se ressembler davantage en partageant un contexte et un système d'acteurs, pour autant, il n'en est rien.

D'un côté, $8^{e} A r t$ qui s'annonce comme un projet d'intérêt général en défendant l'idée d'un public révèle en fait une forte dimension marchande dans le sens où le projet sert de vitrine pour donner de la visibilité à la fois au bailleur et au quartier, reprenant ainsi certaines des thèses au fondement des villes créatives ou villes-évenement (Florida, 2002, Vivant, 2009, Boullier, 2010). Le projet utilise un argumentaire politicien autour de l'urbanité, de la citoyenneté et de la participation, malgré un grand renfort de justifications sur la visée éthique et sur la nécessité de partager le projet, celui-ci peine à exprimer autre chose qu'une volonté de rayonnement et de visibilisation, à la fois des œuvres, du territoire (et du patrimoine immobilier du bailleur) et du bailleur en tant qu'acteur de l'innovation. Cependant ce désir d'être innovant est quelque peu mis à mal par une image traditionnelle donnée d'une commande publique se faisant sans les habitants qui a pour résultat que le projet reste enfermé dans une dimension d'événementiel. Et alors que $8^{e} A r t$ se voulait au service d'un quartier et de ses habitants, le projet se sert de la dimension sociale et citoyenne comme d'une justification.

De l'autre, Prenez Racines! qui se présente comme un projet local sans autre ambition que celle de faire participer les habitants parvient à les mobiliser sur des dimensions complexes (philosophiques, politiques, esthétiques et environnementales) et de ce fait, fait émerger une dimension créative non seulement sur le plan artistique mais aussi sur le plan social et politique. Le projet se veut résolument participatif et traduit les grands enjeux de la politique de la ville, cohésion sociale, recherche de mixité urbaine, accompagnement des populations lors des transformations urbaines et sociales. Toutefois ses enjeux dépassent un simple projet d'animation socioculturelle. Sa portée esthétique assume une complexité et un caractère hybride. Sa portée urbaine va au-delà d'une œuvre dans l'espace public, elle traduit l'intrication de projets issus de champs et de sensibilités différentes et interroge les relations entre ces nouvelles pratiques artistiques et la ville (et inversement) et surtout entre les acteurs de ces pratiques. Enfin sa portée éthique, pose la question de la limite de la participation des habitants et met en lumière la dimension « écosophique » de la ville. Mais le projet est avant 
tout une expérimentation artistique. Il ne se limite donc pas à une fonction altruiste et sociale, il est aussi au service de l'art et de l'artiste en permettant l'expérimentation de pratiques artistiques hybrides dans les quartiers d'habitat social.

Les pratiques artistiques qui sous-tendent ces projets font des quartiers concernés de nouveaux lieux de la créativité, de l'altérité et de la diversité tant artistique que sociale et urbaine, dans le sens où elle permettent aux habitants de s'impliquer dans la mise en œuvre de projets artistiques donnant lieu à des réflexions complexes et portant des enjeux sociaux et politiques mais aussi environnementaux. Elles interrogeant le rôle de l'artiste et sa posture au cœur de la société.

Ces deux exemples montrent comment des projets artistiques menés dans des quartiers de logement social servent de révélateurs de transformations dans les rôles et leurs justifications mais aussi dans les représentations et les imaginaires et leurs limites. Ces projets artistiques sont pour une part présentés comme un service public, dans une logique civique : au service de populations et d'un territoire et véhiculant l'idée d'une démocratie vivante et permanente qui ne soit pas une implication intermittente mais qui voie le citoyen comme acteur. D'autre part, les quartiers d'habitat social travaillés par des pratiques artistiques participatives se montrent comme de nouvelles scènes de créativité, associant militantisme et engagement affectif. Enfin, ces pratiques artistiques fournissent les éléments d'une représentation positive et pacifiée de la ville, une ville faite d'une superposition d'usages, d'invention de nouvelles formes d'être ensemble où la création est au service des habitants. 


\section{Bibliographie}

Madeleine Akrich., Michel Callon, Bruno Latour, Sociologie de la traduction. Textes

fondateurs, Sciences sociales, Presses de l'École des Mines, 2006, 303 pages

Paul Ardenne, Un art contextuel. Création artistique. En milieu urbain. En situation.

D'intervention. De participation, Champs sciences humaines, Editions Flammarion, 2002, 254 pages

Hannah Arendt, Condition de l'homme moderne, Evolution, Editions du Seuil, 2001, 406 pages

Hannah Arendt, Qu'est ce que la politique ?, Points essais, Editions du Seuil, 1995, 215 pages Loïc Blondiaux, Le nouvel esprit de la démocratie. Actualité de la démocratie participative, La république des idées, Seuil, 2008, 109 pages

Hugues Bazin, Espaces populaires de création culturelle. Enjeux d'une recherche-action situationnelle, Cahiers de l'action, INJEP, 2006, 91 pages

Dominique Boullier, La ville événement. Foules et publics urbains, La ville en débats, Presses Universitaires de France, 2010, 147 pages

Nicolas Bourriaud, Esthétique relationnelle, Documents sur l'art, Les presses du réel, 1998, pages

Jean-Paul Fourmentraux, L'œuvre commune affaire d'art et de citoyen, Les presses du réel, 2012, 319 pages

Paulo Freire, Pedagogy of the oppressed, New York continuum, 1997, 192 pages

Sébastien Gazeau, Quartiers. Les projets participatifs au cour de la (politique de la) ville, ARTfactories, AutrepARTs, 2012, 98 pages

Catherine Grout, Pour une réalité publique de l'art, Esthétiques, L'Harmattan, 2000, 315 pages

Félix Guattari, Les trois écologies, La philosophie en effet, Editions Galilée, 1989, 72 pages Jüngen Habermas, L'espace public. Archéologie de la publicité comme dimension constitutive de la société bourgeoise, Critique de la politique, Editions Payot et rivages, 1988, 330 pages

Fabrice Lextrait, Frédéric Kahn, Nouveaux territoires de l'art, éditions Sujet-Objet, 2005, 295 pages 
Françoise Liot (coord.), Projets culturels et participation citoyenne. Le rôle de la médiation et de l'animation en question, Animation et territoires, L'Harmattan, 2010, 221 pages Antonio Negri, Inventer le commun des hommes. Tout étant produit par tous, appartient à tous, Essais, Editions Bayard, 2010, 295 pages

Catherine Neveu, «La citoyenneté entre individuel et collectif. Bref portrait de « jeunes » animateurs issus de l'immigration en citoyens », Ville-Ecole-Intégration, $\mathrm{n}^{\circ} 11$, septembre 1999 , pages $68-80$

Jacques Rancière, Le partage du sensible, éditions la Fabrique, 2000, 74 pages

Christian Ruby, Nouvelles Lettres sur l'éducation esthétique de l'homme, Lettres, La Lettre volée, 2005, 225 pages

Dominique Schnapper, Qu'est que la citoyenneté ?, Folio actuel, Folio, 2000, 320 pages Elsa Vivant, Qu'est ce que la ville créative?, La ville en débat, Presses Universitaires de France, 2009, 89 pages

Joëlle, Outdoor Art. La sculpture et ses lieux, Les empêcheurs de penser en rond, Editions La Découverte, 2013, 237 pages

Joëlle Zask, Participer. Essai sur les formes démocratiques de la participation, Editions Le bord de l'eau, 2011, 326 pages 